\title{
THE REPRESENTATION OF FACTS IN PHYSICAL THEORIES
}

\author{
Hans Primas \\ Laboratory of Physical Chemistry \\ ETH-Zentrum, CH-8092 Zürich, Switzerland
}

\begin{abstract}
The purpose of this contribution is to call attention to a problem which has not received the interest which, in my opinion, it deserves: the problem of representation of facts in physical theories. The crucial point is, that within the framework of fundamental physical theories, the representation of facts requires a breaking of the time-reversal symmetry and nonanticipative measuring instruments. These conditions are satisfied only when the apparatus is described as a system with infinitely many degrees of freedom. In the framework of algebraic quantum theory generalized K-systems can represent facts at least in an asymptotic sense. Such a representation removes the main stumbling block which stands in the way of a fundamental theory of measurement in quantum theory.
\end{abstract}

\section{INTRODUCTION}

By a fact we mean an event in the past which retains its facticity in the future. One crucial precondition for the existence of facts is the possibility to discriminate between the past and the future. This prerequisite is also called "the anisotropy of time" or "the arrow of time". In classical physical theories, we presuppose in addition that any reasonable statement about a fact is either true or false, even if we do not know it. This condition depends on the possibility to distinguish different things in our world. Yet, facts are not restricted to the macroscopic world where distinguishability seems to be no problem. In quantum theory the basic precondition of distinguishability and separability are not automatically justified. If we postulate the universal validity of quantum theory it is no longer evident that facts in the classical sense exist at all. However, it is not necessary to stick to the classical position. It is sufficient to understand instruments which register facts in the sense of Fock (1957):

"We call an 'instrument' such an arrangement which on the one hand can be influenced by, and interact with, an atomic object and on the other hand permits a classical description with an accuracy sufficient for the purpose of registering the said influence (consequently, the handling of the instrument so defined does not need further 'means of observation'). It should be noted at once that in this definition of the instrument it is quite immaterial whether the 'instrument' is made by human hands or represents a natural combination of external conditions suitable for the observation of the micro-object."

The crucial point is that all what is asked for is "an accuracy sufficient for the purpose of registering". So we have to bear in mind the possibility that in quantum theory facts in the classical sense turn up only in limit of long time. In this case we speak of asymptotic facts which manifest themselves after sufficiently long but finite time as approximate facts. It may be that the documents which tell us something about facts can be destroyed or changed. Nevertheless, we require that the facts themselves (whether exact or approximate) cannot be changed by any future influences. 
Since facts never disappear but can in principle always be called back from the past into the present, with every new event the set of all facts increases with time.

The only known way to formulate physical laws in a non-phenomenological manner applies to strictly isolated systems. We call a system strictly isolated if all variables which can influence the system can be taken into account in the specification of its initial (or just as well of its final) state. ${ }^{1}$ Using the space-time concepts of Newtonian mechanics, the basic principles of Hamiltonian mechanics are in every respect time-symmetric so that all fundamental phenomena are symmetrical with respect to an interchange of past and future. That is, the basic equations of motion are invariant under an involution which exchanges the time parameter $t$ by $-t .^{2}$ This invariance is called the time-reversal symmetry.

In the Hamiltonian formalism for strictly isolated systems time is not a property of the system since for such systems the time coordinate can in principle be eliminated without loss of physical content. ${ }^{3}$ For strictly closed systems time is not an observable. Accordingly, on the fundamental level causation cannot be defined in terms of time order or by the idea that a cause is ontologically more basic than its effects. All we have at our disposal are time-symmetric correlations. On the other side, in everyday life the past is knowable and the future is not. Hence we tacitly presuppose a "principle of retarded causality": no effect can precede its cause. But at a fundamental level there is no distinction between past and future. So it makes no sense to speak of cause and effect: fundamental causality is arrowless. ${ }^{4}$

For example the electromagnetic interaction between two electrons cannot be described by a time-directed notion of causality, e. g. by a retarded or an advanced interaction. ${ }^{5}$ In a particle theory, a consistent description has to use the unique symmetrized interaction in which advanced and retarded interactions are combined half and half, thus treating both electrons on an equal footing. ${ }^{6}$

The only systems of interest to experimental science are open. However, there are no fundamental laws for open systems. For example, any dynamical law for an open system contains contextual phenomenological parameters (like relaxation times). In order to discuss open systems from a fundamental viewpoint they must first be combined with all systems which with they interact or are correlated. If we include the whole environment of an open system we can describe the resulting system as a strictly isolated Hamiltonian system by first principles. However, the necessary additional conditions (like initial or boundary conditions) for a description of the open object subsystem are not given by first principles but must be chosen in a way appropriate for the experiments we perform.

1 Compare Havas (1965), p. 348.

2 An involution is an operation whose square is the identity. The involution associated with time-reversal does not only change the direction of time but also associated quantities like the velocity, the momentum, the angular momentum, the electrical current and the magnetic field. In quantum mechanics, the time-reversal is an antilinear involutive operation which changes any complex number into its complex conjugate. In elementary particle physics, the invariant involution associated with time-reversal $T\left(T^{2}=1\right)$ also involves the space reflection $P\left(P^{2}=1\right)$ and the charge conjugation $C\left(C^{2}=1\right)(C P T$-theorem).

3 Using Jacobi's principle of least action, Hamiltonian dynamics can be formulated in a completely geometrical language. Compare e.g. Synge (1960), sections 82-83, pp. 136-139.

4 Compare also Costa de Beauregard (1987), p. 134.

5 Already in the year 1909 Einstein (1909) argued that there are no fundamental reasons to rule out timebackward advanced solutions.

6 Compare also the example discussed by Feynman \& Hibbs (1964), p. 251. 
In everyday life there is an intrinsic dissimilarity of the past and the future. This historical nature of the world is an precondition of all engineering science. In engineering physics the direction of causation is always assumed to go from past to future. That is, in order to derive engineering physics from fundamental physics, the time-reversal symmetry of fundamental physics has to be broken. The first difficulty one encounters in carrying out such a program is technical. Although the phenomenon of spontaneous symmetry breaking is well-understood in modern physical theories, it poses formidable mathematical problems. The second difficulty refers to a conceptually deep problem. If the time-reversal symmetry is broken one gets two representations, one satisfying the generally accepted rules of retarded causality and the other one the strange rules of advanced causality. Advanced causality is a conceptual possibility which is not banned by any fundamental physical law. The usual choice of retarded causality cannot be explained by a statistical mechanical formulation of the "second law" without an a priori postulate imposing an asymmetric evolution toward increasing time.

In this contribution I will not discuss any reasons for selecting the retarded representation but concentrate on a proper description of open physical systems which can represent facts. This is not a trivial task - not even in classical physics. In section 2 we recapitulate well-known tools from engineering physics which are necessary for the description of facts in classical physics. In section 3 we discuss the additional difficulties which arise in quantum theory. We give a short outline of the problems which are related to the so-called measurement problem. The concepts used in engineering physics for the description of nonanticipative input-output systems are instrumental for the representation of facts by asymptotically disjoint states.

\section{THE REPRESENTATION OF FACTS IN CLASSICAL PHYSICS}

\section{I EVERY LABORATORY INSTRUMENT IS NONANTICIPATIVE}

Laboratory phenomena are commonly described in terms of cause-and-effect relationships. An input-output system is a mathematical description of an experimental stimulus-response relationship by a dynamical system which, when subjected to the same stimuli, yields the same response as the experimental object. Such an input-output system may be regarded as an abstract operator $\mathscr{R}$ which transforms an input signal $t \mapsto y(t)$ into an output signal $t \mapsto x(t)$ by $x=\mathscr{R}\{y\}$. If this functional relationship is continuous, the response operator $\mathscr{R}$ can be represented by a Volterra expansion

$$
x(t)=R^{(0)}(t)+\int_{-\infty}^{\infty} d s R^{(1)}(t, s) y(s)+\frac{1}{2} \int_{-\infty}^{\infty} d s \int_{-\infty}^{\infty} d s^{\prime} R^{(2)}\left(t, s, s^{\prime}\right) y(s) y\left(s^{\prime}\right)+\cdots .
$$

If the input and the output are real-valued functions, the integral kernel $R^{(1)}: \mathbb{R}^{2} \rightarrow \mathbb{R}$ is the linear response function, the kernel $R^{(2)}: \mathbb{R}^{3} \rightarrow \mathbb{R}$ the quadratic response function, etc. However, not every input-output system of this kind can be realized with a physical instrument in real time. In the world of the experimenter there is a preferred direction of time. Therefore the set of all temporal instants must be ordered such that the past precedes the future. The crucial restriction for a dynamical system representing experimental data in real time is: "No output can occur before the input". More precisely, this condition implies

$$
R^{(1)}(t, s)=0 \quad \text { for } s>t \quad, \quad R^{(2)}\left(t, s, s^{\prime}\right)=0 \text { for } s>t \text { or } s^{\prime}>t, \cdots,
$$


so that

$$
x(t)=R^{(0)}(t)+\int_{-\infty}^{t} d s R^{(1)}(t, s) y(s)+\frac{1}{2} \int_{-\infty}^{t} d s \int_{-\infty}^{t} d s^{\prime} R^{(2)}\left(t, s, s^{\prime}\right) y(s) y\left(s^{\prime}\right)+\cdots .
$$

In such a system, changes of the output cannot anticipate changes of the input. In the terminology of the physicists and engineers such input-output systems are called "causal systems". Since in the philosophical literature the notion of "causality" is used differently, I shall avoid this terminology and speak of "nonanticipative systems".

For a given input-output system, the kernels $R^{(0)}, R^{(1)}, R^{(2)}, \ldots$ can be determined individually by appropriate experiments. In particular, the linear response function $R^{(1)}$ is well-defined for every nonlinear system. For simplicity we restrict our discussion to the linear response. We consider a continuous, linear nonanticipative system with the linear response function $R^{(1)}$

$$
x(t)=R^{(0)}(t)+\int_{-\infty}^{t} R^{(1)}(t, s) y(s) d s .
$$

This input-output map does, however, not characterize the linear system completely. A proper description requires additionally conditions that warrant the controllability and constructibility of the states of the linear system. ${ }^{7}$ Such conditions are automatically fulfilled for systems which are invariant under time-translations. In this case we have $d R^{(0)}(t) / d t=0$ and $R^{(1)}(t, s)=R(t-s)$. Without restricting the generality, we may neglect the trivial constant $R^{(0)}$ so that a continuous, time-invariant, linear system can be described by

$$
x(t)=\int_{-\infty}^{\infty} R(t-s) y(s) d s
$$

The function $R: \mathbb{R} \rightarrow \mathbb{R}$ is called the linear response of the system. If the system is nonanticipative, the linear response function fulfills the condition

$$
R(t)=0 \text { for } t<0 .
$$

The Fourier transform of the linear response function is called the frequency-response function $H: \mathbb{R} \rightarrow \mathbb{C}$

$$
H(\lambda):=\int_{-\infty}^{\infty} e^{i \lambda t} R(t) d t=\int_{0}^{\infty} e^{i \lambda t} R(t) d t \quad, \quad \lambda \in \mathbb{R}
$$

In electrical network theory, one defines a so-called transfer function as the Fourier-Laplace transform $\mathrm{H}^{+}: \mathbb{R} \rightarrow \mathbb{C}$ of the linear response function,

$$
H^{+}(\lambda+i p):=\int_{0}^{\infty} e^{i \lambda t} e^{-p t} R(t) d t \quad, \quad \lambda \in \mathbb{R}, \quad p>0 .
$$

The transfer function $z \mapsto H^{+}(z)$ is the analytic continuation of the frequency-response function $\lambda \mapsto H(\lambda)$, it is holomorphic in the open upper half-plane $\mathbb{C}^{+}:=\{z \in \mathbb{C} \mid \Im(z)>0\}$. The frequency-response function is the boundary value of the transfer function:

7 Compare for example Kalman, Falb \& Arbib (1969), in particular pp. 324-325. 


$$
H(\lambda)=\lim _{p \downarrow 0} H^{+}(\lambda+i p) \quad, \quad \lambda \in \mathbb{R}, \quad p>0
$$

The analyticity of the transfer function reflects the nonanticipative behavior of a time-invariant linear system.

In network theory, the function $A: \mathbb{R} \rightarrow \mathbb{R}^{+}$with $A(\lambda):=|H(\lambda)|^{2}$ is referred to as the amplitude characteristics. Given a certain amplitude characteristic $A$, the important engineering question arises whether or not a filter with this amplitude characteristics can be realized by a nonanticipative linear dynamical system. In other words: Does there exist a frequency-response function $\lambda \mapsto H(\lambda)$ such that

$$
A(\lambda):=|H(\lambda)|^{2} \quad \text { with } \int_{-\infty}^{\infty} e^{-i \lambda t} H(\lambda) d \lambda=0 \text { for } t<0 \quad \text { ? }
$$

The answer is given by the Paley-Wiener criterion: ${ }^{8}$

Necessary and sufficient for a square integrable amplitude function $A: \mathbb{R} \rightarrow \mathbb{R}^{+}$to be realizable by a nonanticipative linear dynamical system with a response function $R: \mathbb{R} \rightarrow \mathbb{R}$

$$
R(t)=0 \text { for } t<0, \quad A(\lambda)=\left|\int_{0}^{\infty} e^{i \lambda t} R(t) d t\right|^{2},
$$

is the Paley-Wiener criterion

$$
\int_{-\infty}^{\infty} \frac{|\ln A(\lambda)|}{1+\lambda^{2}} d \lambda<\infty
$$

If the Paley-Wiener criterion is satisfied, one can factor the square of the amplitude characteristic $A$ on the real line into a product of two functions which have holomorphic extensions into complex half-planes. Therefore we can write

$$
A(\lambda)=H(\lambda) H(\lambda)^{*} \quad, \quad \lambda \in \mathbb{R},
$$

where $\lambda \mapsto H(\lambda)$ is the boundary value of an analytic function $z \mapsto H^{+}(z)$, holomorphic in the open upper half-plane $\mathbb{C}^{+}:=\{z \in \mathbb{C} \mid \Im(z)>0\}$. Accordingly, $\lambda \mapsto H(\lambda)^{*}$ is the boundary value of another analytic function $z \mapsto H^{-}(z)$, holomorphic in the open lower half-plane $\mathbb{C}^{-}:=\{z \in \mathbb{C} \mid \Im(z)<0\}$,

$$
H(\lambda):=\lim _{p \downarrow 0} H^{+}(\lambda+i p) \quad, \quad H(\lambda)^{*}:=\lim _{p \downarrow 0} H^{-}(\lambda-i p) \quad, \quad p>0 \quad, \quad \lambda \in \mathbb{R} .
$$

The function $\lambda \mapsto H(\lambda)$ is the frequency-response function of a past-determined nonanticipative linear system, while the function $\lambda \mapsto H(\lambda)^{*}$ corresponds to the frequency-response function of a future-determined anticipative linear system.

Since $|H(\lambda)| \in L^{2}(\mathbb{R})$, the unique extension $H^{+}$is in the Hardy class $H^{2}\left(\mathbb{C}^{+}\right)$and therefore admits a unique outer-inner factorization

$$
H^{+}(z)=\Phi(z) \Psi(z) \quad, \quad z \in \mathbb{C}^{+} .
$$

The outer function $\Phi$ is uniquely given by

8 Paley \& Wiener (1934), pp. 16-17. The condition of square integrability in the theorem by Paley and Wiener is not essential. It can be replaced by much weaker conditions, for example by the requirement that the amplitude function is a tempered generalized function. Compare Pfaffelhuber (1971). 


$$
\Phi(z)=\exp \frac{1}{\pi i} \int_{-\infty}^{\infty} \frac{1+\lambda z}{\lambda-z} \frac{|\ln H(\lambda)|}{1+\lambda^{2}} d \lambda \quad, \quad z \in \mathbb{C}^{+}
$$

There is also a unique representation for an inner function $z \mapsto \Psi(z)\left(z \in \mathbb{C}^{+}\right)$whose boundary value $\omega \mapsto \Psi(\omega)(\omega \in \mathbb{R})$ represents an all-pass filter with constant amplitude which causes only additional phase delay. In case $\Psi(z)=1$, the filter is said to be of minimum phase type. It has the frequency-response function $H(\omega)=\Phi(\omega+i 0)(\omega \in \mathbb{R})$.

To summarize: The appropriate tools for the discussion of nonanticipative laboratory instruments are the theory of Hardy spaces, the Paley-Wiener criterion and the Wiener factorization. In particular, it follows that a nonanticipative linear filter of minimum phase type is uniquely given by its amplitude characteristic.

\subsection{DETERMINISTIC AND NONDETERMINISTIC PROCESSES}

The laws of any Hamiltonian mechanics are invariant under time-reversal. In particular the dynamics is both forward deterministic and backward deterministic. A present state determines uniquely the future and the past states so that in principle exact prediction and exact retrodiction are possible. Using only mechanical tools it is therefore impossible to distinguish between cause and effect. This can only be achieved by temporally one-sided processes. Dissipative stochastic processes are examples for one-sided processes. They are backward-deterministic and forwardindeterministic, they can be retrodicted exactly but predictions are at best probabilistic.

The paradigmatic example for a backward-deterministic and forward-nondeterministic process is the Wiener process, a mathematically rigorous model for the idealized Brownian motion. In his model, Norbert Wiener (1923) proved Perrin's conjecture that all paths of an idealized Brownian motion are almost certainly (i.e. with probability one) continuous but nowhere differentiable. That is, in Wiener's idealization, a Brownian path consists entirely of sharp corners.

Wiener's work initiated the mathematical theory of stochastic processes and functional integration. Ten years later, Kolmogoroff (1933) laid the foundation for the modern axiomatic treatment of mathematical probability theory in terms of measure theory. However, it would be mistaken to believe that the theory of stochastic processes in the sense of Kolmogorov has superseded Wiener's ideas. In his work on generalized harmonic analysis during 1925-1930, Wiener (1930) based his theory not on equivalence classes of Lebesgue square integrable functions but on individual measurable functions $t \mapsto f(t)$ for which the individual autocorrelation function

$$
t \mapsto A_{f}(t):=\lim _{T \rightarrow \infty} \frac{1}{2 T} \int_{-T}^{+T} f(\tau)^{*} f(t+\tau) d \tau
$$

exists for all $t \in \mathbb{R}$. Writing $A_{f}$ as Fourier transform

$$
A_{f}(t)=\int_{-\infty}^{\infty} e^{i \lambda t} d S_{f}(\lambda)
$$

he obtained what is now called the individual spectral distribution function $S_{f}$.

These relations have their counterparts in Kolmogorov's ensemble theory of stationary stochastic processes. A complex-valued stochastic process $\{f(t \mid \boldsymbol{\omega}) \mid t \in \mathbb{R}, \boldsymbol{\omega} \in \boldsymbol{\Omega}\}$ in the sense of Kolmogorov is a family of complex-valued random variables $\boldsymbol{\omega} \mapsto f(\bullet \mid \boldsymbol{\omega})$ on a common Kolmogorov probability space $(\boldsymbol{\Omega}, \boldsymbol{\Sigma}, \boldsymbol{\mu})$, where $\boldsymbol{\Omega}$ is a set, $\boldsymbol{\Sigma}$ a $\sigma$-algebra, and $\boldsymbol{\mu}$ a probability measure. For 
a fixed $\boldsymbol{\omega} \in \boldsymbol{\Omega}$, the function $t \mapsto f(t \mid \boldsymbol{\omega})$ is a complex-valued function, called a trajectory (or a realization) of the stochastic process $\{f(t \mid \boldsymbol{\omega}) \mid t \in \mathbb{R}, \boldsymbol{\omega} \in \boldsymbol{\Omega}\}$ corresponding to the event $\boldsymbol{\omega}$. A stochastic process is an equivalence class of trajectories with the same family of joint probability densities. The mean value $m_{f}$ and the covariance function $C_{f}$ of the stochastic process $\{f(t \mid \boldsymbol{\omega}) \mid t \in \mathbb{R}, \boldsymbol{\omega} \in \boldsymbol{\Omega}\}$ are defined by

$$
\begin{gathered}
m_{f}(t):=\mathcal{E}\{f(t \mid \cdot)\}:=\int_{\boldsymbol{\Omega}} f(t \mid \boldsymbol{\omega}) \boldsymbol{\mu}(d \boldsymbol{\omega}), \\
C_{f}(t, s):=\mathcal{E}\left\{f(t \mid \cdot)^{*} f(s \mid \cdot)\right\}-m_{f}(t)^{*} m_{f}(s)=\int_{\boldsymbol{\Omega}} f(t \mid \boldsymbol{\omega})^{*} f(s \mid \boldsymbol{\omega}) \boldsymbol{\mu}(d \boldsymbol{\omega})-m_{f}(t)^{*} m_{f}(s) .
\end{gathered}
$$

A stochastic process is said to be stationary if all joint probability densities are invariant under time translation. In this case, the mean value is time-independent, while the covariance function depends only on the difference of two times,

$$
C_{f}(t, s)=C_{f}(t-s) \text { for all } t, s \in \mathbb{R} .
$$

Khintchine (1934) proved that the covariance function of every stationary stochastic process can be represented in the form

$$
C_{f}(t)=\int_{-\infty}^{\infty} e^{i \lambda t} d F_{f}(\lambda)
$$

where $F_{f}: \mathbb{R} \rightarrow \mathbb{R}$ is a real, never decreasing and bounded function, called the spectral distribution function of the stochastic process.

For ergodic stationary stochastic processes Wiener's analytical representation theorem for a single function follows from Khintchine's ensemble representation theorem for stochastic processes: A realization $t \mapsto f(t \mid \boldsymbol{\omega})$ ( $\boldsymbol{\omega}$ fixed) of an ergodic stationary stochastic process $\{f(t \mid \boldsymbol{\omega}) \mid t \in \mathbb{R}, \boldsymbol{\omega} \in \boldsymbol{\Omega}\}$ will be, with probability one, such a function that Wiener's individual autocorrelation function $t \mapsto A_{f}(t \mid \boldsymbol{\omega})$,

$$
A_{f}(t \mid \boldsymbol{\omega}):=\lim _{T \rightarrow \infty} \frac{1}{2 T} \int_{-T}^{+T} f(\tau \mid \boldsymbol{\omega})^{*} f(t+\tau \mid \boldsymbol{\omega}) d \tau \quad, \quad \boldsymbol{\omega} \text { fixed , }
$$

exists and is equal to the covariance function $t \mapsto C_{f}(t)$. But it is important to realize that Wiener's theory is in no way probabilistic but applies to single well defined functions rather than to an ensemble of functions. ${ }^{9}$

Let $\boldsymbol{\Sigma}(a, b) \subset \boldsymbol{\Sigma}$ be the $\sigma$-field generated by the stochastic process $t \mapsto f_{t}:=f(t \mid \cdot)$ in the time interval $a \leq t \leq b$, so that $\boldsymbol{\Sigma}(-\infty,+\infty)=\boldsymbol{\Sigma}$. Since $\{\boldsymbol{\Sigma}(-\infty, t) \mid t \in \mathbb{R}\}$ is a monotonically increasing, and $\{\boldsymbol{\Sigma}(t,+\infty) \mid t \in \mathbb{R}\}$ a monotonically decreasing family of $\sigma$-fields, the remote past $\boldsymbol{\Sigma}(-\infty)$ and the remote future $\boldsymbol{\Sigma}(+\infty)$ are given by

$$
\boldsymbol{\Sigma}(-\infty):=\bigcap_{t \leq 0} \boldsymbol{\Sigma}(-\infty, t) \quad, \quad \boldsymbol{\Sigma}(+\infty):=\bigcap_{t \leq 0} \boldsymbol{\Sigma}(t,+\infty)
$$

9 In his later work on Extrapolation, Interpolation, and Smoothing of Stationary Time Series, Wiener (1949) used individual functions $t \mapsto f(t)$, and not equivalence classes $t \mapsto\{f(t \mid \boldsymbol{\omega}) \mid \boldsymbol{\omega} \in \boldsymbol{\Omega}\}$. This approach has been criticized as unnecessarily cumbersome (Kakutani (1950)). However, it has to be stressed that for the prediction of an individual time series only Wiener's approach is conceptually sound - for weather prediction or antiaircraft fire control there is no ensemble of trajectories but just a single individual trajectory from whose past behavior one would like to predict something about its future behavior. 
These concepts are important for the prediction and the retrodiction of stochastic processes. Fist we consider the problem of prediction. Let $\mathscr{L}_{t}^{2}$ be the Hilbert space consisting of all $\mathbf{\Sigma}(-\infty, t)$. measurable functions that are square-integrable with respect to the probability measure $\boldsymbol{\mu}$, $\mathscr{L}_{t}^{2}:=L^{2}\{\boldsymbol{\Omega}, \boldsymbol{\Sigma}(-\infty, t), \boldsymbol{\mu}\}$. Having observed the past $\left\{f_{s} \mid s \leq t\right\}$ of a process, one wants to forecast $\tau \mapsto f_{t+\tau}$ using an element $g_{t, \tau}$ of $\mathscr{L}_{t}^{2}$. The predictor $g_{t, \tau}$ is in general a nonlinear function of the observed process $\left\{f_{s} \mid s \leq t\right\}$. If one adopts the least-square criterion, the prediction error is given by:

$$
\sigma^{2}(t, \tau)=\mathcal{E}\left\{\left|f_{t+\tau}-g_{t, \tau}\right|^{2}\right\} .
$$

Since the process is assumed to be stationary, the error for the optimum predictor does not depend on $t, \sigma(t, \tau)=\sigma(\tau)$. The error for the optimal mean-square predictor is the conditional expectation of the process, given $\mathbf{\Sigma}(-\infty, t),{ }^{10}$

$$
g_{t, \tau}^{\mathrm{opt}}=\mathcal{E}\left\{f_{t+\tau} \mid \boldsymbol{\Sigma}(-\infty, t)\right\} .
$$

A process $\left\{f_{t} \mid t \in \mathbb{R}\right\}$ is called forward deterministic if the optimal predictor in terms of the past $\mathbf{\Sigma}(-\infty, 0)$ allows an error-free prediction. In this case the process is in fact already determined by the remote past $\mathbf{\Sigma}(-\infty)$, and a perfect prediction can even be performed by a constructive algorithm. ${ }^{11}$ If an error-free prediction is not possible, the process is called forward nondeterministic. Every process can be represented uniquely as the sum of a forward deterministic process and a socalled forward purely nondeterministic process (where, of course, one component may be absent). A process is called forward purely nondeterministic if the unconditional expectation is the best forecast. In this case the remote past $\boldsymbol{\Sigma}(-\infty)$ is the trivial Borel field $\{\varnothing, \boldsymbol{\Omega}\}$ consisting only of the impossible event $\varnothing$ and the certain event $\boldsymbol{\Omega}$. The present state of a forward deterministic process determines all its future states, while a forward purely nondeterministic process contains no components that can be predicted exactly from an arbitrarily long past record.

There is another extrapolation problem: retrodiction. Given the trajectory of a stochastic process on the positive real axis, can we retrodict the behavior of the process on the negative real axis? Of course, the answer is analogous to the problem of prediction; formally, $t$ has just to be replaced by $-t$. Therefore we arrive at the following classification: ${ }^{12}$

A process $\left\{f_{t} \mid t \in \mathbb{R}\right\}$ is called

- forward deterministic if

- backward deterministic if

$$
\begin{aligned}
& \boldsymbol{\Sigma}(-\infty)=\mathbf{\Sigma}, \\
& \boldsymbol{\Sigma}(+\infty)=\boldsymbol{\Sigma}, \\
& \boldsymbol{\Sigma}(-\infty)=\boldsymbol{\Sigma}(+\infty)=\boldsymbol{\Sigma}, \\
& \boldsymbol{\Sigma}(-\infty)=\{\varnothing, \boldsymbol{\Omega}\}, \\
& \boldsymbol{\Sigma}(+\infty)=\{\varnothing, \boldsymbol{\Omega}\} .
\end{aligned}
$$$$
\text { - bidirectionally deterministic if }
$$$$
\text { - forward purely nondeterministic if }
$$

- backward purely nondeterministic if

For a forward purely nondeterministic process the remote past does not contain any information that could be useful for predictions. For backward purely nondeterministic processes the remote future does not contain any information that could be useful for retrodictions. Bidirectional deterministic processes are forward and backward deterministic, they correspond to deterministic motions of time-reflection invariant Hamiltonian mechanics. It is important that in general forward determinism does not imply backward determinism. In fact, there exist stationary processes

10 Compare for example Rosenblatt (1971), p. 164.

11 Compare Scarpellini (1979); Scarpellini (1979).

12 Compare Krengel (1971), Krengel (1973). 
which are forward purely nondeterministic and backward deterministic, and stationary processes which are forward deterministic and backward purely nondeterministic.

By breaking the time-reversal symmetry of the Hamiltonian dynamics one can derive one-sided processes. However, the spontaneous breaking of the symmetry of a group of order two gives two elementary realizations which have the same logical status. That is, if it is possible to derive backward deterministic and forward purely nondeterministic processes, then it is also possible to derive forward deterministic and backward purely nondeterministic processes. The decision which of the two possibilities is appropriate can therefore not come from the first principles of physics. So the conceptual problem is not the breaking of the time-reversal symmetry (though this may pose difficult mathematical questions), but the proper selection of one or the other one-sided realization.

To summarize: The theory of stochastic processes allows a precise description of all processes resulting from the breaking the time-reversal symmetry of a classical Hamiltonian system. Every process can be decomposed in a forward deterministic and a forward purely nondeterministic process. Since forward determinism does not imply backward determinism, a process can also be decomposed in a backward deterministic and a backward purely nondeterministic process. There exist mathematical models for all combinations of forward and backward determinism, and of forward and backward pure nondeterminism.

\subsection{ForWARD PURELY NONDETERMINISTIC PROCESSES GENERATE CLASSICAL K-FLOWS}

A flow $\left\{\boldsymbol{\tau}_{t} \mid t \in \mathbb{R}\right\}$ on a probability space $(\boldsymbol{\Omega}, \boldsymbol{\Sigma}, \boldsymbol{\mu})$ is said to be a K-flow ${ }^{13}$ if there exists a $\sigma$-subalgebra of measurable sets $\boldsymbol{\Sigma}_{0} \subset \boldsymbol{\Sigma}$ such that for $\boldsymbol{\Sigma}_{t}:=\boldsymbol{\tau}_{t} \boldsymbol{\Sigma}_{0}$ the following conditions hold: 14

- $\boldsymbol{\Sigma}_{0} \subset \boldsymbol{\Sigma}_{t}$ for every $t>0$,

- $\vee^{\infty} \boldsymbol{\Sigma}_{t}=\boldsymbol{\Sigma}$,

$t=-\infty$

- $\bigwedge_{t=-\infty} \boldsymbol{\Sigma}_{t}$ is the trivial $\sigma$-algebra consisting of the sets of measure 0 and 1 .

Here $\vee$ is the lattice sum and $\wedge$ is the intersection. Every $\mathrm{K}$-flow is ergodic and has the mixing property of every degree. If we define $\boldsymbol{\Sigma}_{0}:=\boldsymbol{\Sigma}(-\infty, 0)$, every forward purely nondeterministic stationary process $\left\{f\left(\boldsymbol{\tau}_{t} \boldsymbol{\omega}\right) \mid t \in \mathbb{R}, \boldsymbol{\omega} \in \boldsymbol{\Omega}\right\}$ on a probability space $(\boldsymbol{\Omega}, \boldsymbol{\Sigma}, \boldsymbol{\mu})$ generates a K-flow.

Every classical K-flow can be represented algebraically as a dynamical $\mathrm{W}^{*}$-system. The dynamical system $\left(\boldsymbol{\Omega}, \boldsymbol{\Sigma}, \boldsymbol{\mu}, \boldsymbol{\tau}_{t}\right)$ corresponds to the dynamical W*-system $\left(\mathscr{M}, \rho, \alpha_{t}\right)$ with the commutative $W^{*}$-algebra $\mathscr{M}=L^{\infty}(\boldsymbol{\Omega}, \boldsymbol{\mu})$. The automorphism group $\left\{\alpha_{t} \mid t \in \mathbb{R}\right\}$ on $L^{\infty}(\boldsymbol{\Omega}, \boldsymbol{\mu})$ is defined by $\alpha_{t}\{A(\boldsymbol{\omega})\}=A\left(\boldsymbol{\tau}_{t} \boldsymbol{\omega}\right)$ for all $A \in L^{\infty}(\boldsymbol{\Omega}, \boldsymbol{\mu})$. Let $P_{0}$ be the projection operator from $L^{2}\{\boldsymbol{\Omega}, \boldsymbol{\Sigma}(-\infty,+\infty), \boldsymbol{\mu}\}$ onto $L^{2}\{\boldsymbol{\Omega}, \boldsymbol{\Sigma}(-\infty, 0), \boldsymbol{\mu}\}$ and define a $\mathrm{W}^{*}$-algebra $\mathscr{M}_{0} \subset \mathscr{M}$ by $\mathscr{M}_{0}:=P_{0} \mathscr{M} P_{0}$. Then the K-flow generated by a forward purely nondeterministic stationary process is characterized by the $\mathrm{W}^{*}$-algebras $\mathscr{M}_{t}:=\alpha_{t} \mathscr{M}_{0}$

- $\mathscr{M}_{t} \subset \mathscr{M}_{s}$ for every $t<s$,

$\infty$

- $\quad \vee \mathscr{H}_{t}=\mathscr{M}$,

$t=-\infty$

13 Compare for example Cornfeld, Fomin \& Sinai (1982), p. 280.

14 Compare for example Cornfeld, Fomin \& Sinai (1982), p. 280. 
- $\bigwedge_{t=-\infty}^{\infty} \mathscr{M}_{t}=\mathbb{C} 1$.

Here $\vee \mathscr{M}_{t}$ is the smallest $\mathrm{W}^{*}$-subalgebra of $\mathscr{M}$ which contains all $\mathscr{M}_{t}$, while $\wedge \mathscr{M}_{t}$ is the largest $\mathrm{W}^{*}$-subalgebra of $\mathscr{M}$ which is contained in all $\mathscr{M}_{t}$.

To summarize: Every forward purely nondeterministic stationary process generates a classical Kflow which can be represented as a commutative dynamical $\mathrm{W}^{*}$-system.

\subsection{LINEAR PREDICTION}

Since no systematic approach for nonlinear predictions for forward nondeterministic processes has been established so far, the discussion will be restricted to the linear case. As long as linearity is retained, prediction theory is fairly complete. ${ }^{15}$ This theory refers to weakly stationary processes which are characterized by their first and second moments. A complex-valued process $\left\{f_{t} \mid t \in \mathbb{R}\right\}$ is said to be weakly stationary if the moments up to the second order exist and are stationary, i.e. if for every $t, s, \tau \in \mathbb{R}$ we have

$$
\mathcal{E}\left\{\left|f_{t}\right|^{2}\right\}<\infty \quad, \quad \mathcal{E}\left\{f_{t+\tau}\right\}=\mathcal{E}\left\{f_{t}\right\} \quad, \quad \mathcal{E}\left\{f_{t+\tau}^{*} f_{s+\tau}\right\}=\mathcal{E}\left\{f_{t}^{*} f_{s}\right\} .
$$

Linear prediction theory is based on the Hilbert space $\mathscr{H}_{t}$ spanned by a weakly stationary process $\left\{f_{s} \mid-\infty<s \leq t\right\}$. The Hilbert space $\mathscr{L}_{t}^{2}:=L^{2}\{\boldsymbol{\Omega}, \boldsymbol{\Sigma}(-\infty, t), \boldsymbol{\mu}\}$ is in general much larger than the Hilbert space $\mathscr{H}_{t}$. When the nonlinear predictor $g_{t, \tau}$ is restricted to the subspace $\mathscr{H}_{t} \subseteq \mathscr{L}_{t}^{2}$, one speaks of a linear predictor. For weakly stationary Gaussian processes we have $\mathscr{H}_{t}=\mathscr{L}_{t}^{2}$ so that for Gaussian processes the best predictor is linear. A weakly stationary second-order process is said to be forward deterministic in the linear sense if the least-square prediction error vanishes for the optimum linear predictor.

In spite of the fact that a purely nondeterministic stochastic processes exhibits irreversible and dissipative behaviour, it can be generated by an intrinsically conservative and reversible mechanical model. For example, it is well known that every weakly stationary Gaussian process can be generated as the output of a linear Hamiltonian system with an infinite-dimensional phase space. ${ }^{16}$

For every weakly stationary complex-valued process there exists a unique orthogonal decomposition into a forward deterministic process (in the linear sense) and a forward purely nondeterministic process (in the linear sense). ${ }^{17}$ Let $\mathscr{H}$ be the subspace of $L^{2}\{\boldsymbol{\Omega}, \boldsymbol{\Sigma}, \boldsymbol{\mu}\}$ spanned by the variables $f_{t}$ for all $t \in \mathbb{R}$ and the constant function 1 . The Hilbert space spanned by the process $\left\{f_{s}-m \mid s \leq t\right\}$ will be denoted by $\mathscr{C}_{t}$. Furthermore we define

$$
\mathscr{H}_{-\infty}:=\bigcap_{t \in \mathbb{R}} \mathscr{H}_{t},
$$

and the orthogonal projection $P_{-\infty}$ from $\mathscr{C}$ onto $\mathscr{C}_{-\infty}, \mathscr{H}_{-\infty}=P_{-\infty} \mathscr{H}$. Then the Hilbert space $\mathscr{H}_{t}$ spanned by the stationary process $\left\{f_{s} \mid-\infty<s \leq t\right\}$ decomposes into a direct sum $\mathscr{H}_{t}=\mathbb{C} \oplus \mathscr{H}_{t}^{\mathrm{d}} \oplus \mathscr{H}_{t}^{\text {nd }}$, where $\mathscr{H}_{t}^{\mathrm{d}}$ is the Hilbert space spanned by $\left\{f_{s}^{\mathrm{d}} \mid-\infty<s \leq t\right\}$, where

$$
f_{s}^{\mathrm{d}}:=P_{-\infty}\left\{f_{s}-m\right\} \text {. }
$$

15 For an elementary introduction, compare for example Cramér \& Leadbetter (1967), sect. 5.7 and 7.9.

16 Compare for example Picci (1986). Picci (1988).

17 This decomposition is due to Wold (1938) for the special case of discrete-time stationary processes, and to Hanner (1950) for the case of continuous-time processes. The general decomposition theorem is due to Cramér (1961); Cramér (1961). 
The Hilbert space $\mathscr{H}_{t}^{\text {nd }}$ is spanned by $\left\{f_{s}^{\text {nd }} \mid-\infty<s \leq t\right\}$, where

$$
f_{s}^{\text {nd }}:=\left(1-P_{-\infty}\right)\left\{f_{s}-m\right\} \text {. }
$$

According to a criterion by Wiener and Krein ${ }^{18}$, a weakly stationary complex-valued process $\left\{f_{t} \mid t \in \mathbb{R}\right\}$ with the spectral distribution function $\lambda \mapsto F_{f}(\lambda)$ is purely nondeterministic in the linear sense if and only if the spectral distribution function is absolutely continuous and if

$$
\int_{-\infty}^{\infty} \frac{\left|\ln \left\{\rho_{f}(\lambda)\right\}\right|}{1+\lambda^{2}} d \lambda<\infty,
$$

where $\lambda \mapsto \rho_{f}(\lambda)$ is the positive spectral density,

$$
\rho_{f}(\lambda):=\frac{d F_{f}(\lambda)}{d \lambda}
$$

To summarize: A weakly stationary process is purely nondeterministic in the linear sense if and only if the spectral distribution function is absolutely continuous and satisfies the Paley-WienerKrein criterion. Such processes can be generated by classical Hamiltonian systems which are invariant under time-reversal if and only if the associated phase space is infinite-dimensional.

\subsection{PURELY NONDETERMinistic PROCESSES AND TIME OPERATOR}

Let $\left\{f_{s} \mid t \in \mathbb{R}\right\}$ be a continuous weakly stationary purely nondeterministic process on the probability space $(\boldsymbol{\Omega}, \boldsymbol{\Sigma}, \boldsymbol{\mu})$. Let $\mathscr{C}$ be the subspace of $L^{2}\{\boldsymbol{\Omega}, \boldsymbol{\Sigma}, \boldsymbol{\mu}\}$ spanned by the variables $f_{t}$ for all $t \in \mathbb{R}$. The time evolution for this process is given by a one-parameter group of unitary shift operator $U_{t}$ acting in the Hilbert space $\mathscr{H}$. They are defined by

$$
f_{t+s}=U_{t} f_{s}, f_{t+s}, f_{s} \in \mathscr{H} \quad, \quad t, s \in \mathbb{R} .
$$

The concept of a time operator for weakly stationary purely nondeterministic processes has been introduced by Tjøstheim (1975). ${ }^{19}$ Let $\mathscr{H}_{t}$ be the closed subspace of $\mathscr{H}$ spanned by $\left\{f_{s}^{\text {nd }} \mid-\infty<s \leq t\right\}$. Denote by $P_{t}$ the projection operator from $\mathscr{H}$ onto $\mathscr{H}_{t}$. The set $\left\{P_{t} \mid t \in \mathbb{R}\right\}$ is a spectral family with $P_{s} \leq P_{t}$ for $s<t, P_{-\infty}=0$ and $P_{+\infty}=1$. Then the selfadjoint operator

$$
T:=\int_{-\infty}^{\infty} t d P_{t}
$$

is called the time operator of the purely nondeterministic processes $\left\{f_{s} \mid t \in \mathbb{R}\right\}$. It is shifted by $t$ under the dynamics,

$$
U_{t}^{*} T U_{t}=T+t 1, t \in \mathbb{R} .
$$

The unitary group $\left\{U_{t} \mid t \in \mathbb{R}\right\}$ can be represented by a selfadjoint generator $\Lambda$ as $U_{t}=e^{i t \Lambda}$. If we introduce another unitary group $\left\{V_{\lambda} \mid \lambda \in \mathbb{R}\right\}$ by $V_{\lambda}=e^{-i \lambda T}$, then the unitary operators $U_{t}$ and $V_{\lambda}$ satisfy Weyl's canonical commutation relation

$$
V_{\lambda} U_{t}=e^{i \lambda t} U_{t} V_{\lambda} \quad, \quad \lambda, t \in \mathbb{R} .
$$

On an appropriate domain, the time operator $T$ and the generator $\Lambda$ satisfy Heisenberg's canonical commutation relations

18 Wiener (1942), republished as Wiener (1949); Krein (1945), Krein (1945).

19 Compare allso Hanner (1950), Tjøstheim (1976a), Tjøstheim (1976b), Gustavson \& Misra (1976). 


$$
T \Lambda-\Lambda T=i 1 \text {. }
$$

The dynamical system associated with the weakly stationary purely nondeterministic process $\left\{f_{s} \mid t \in \mathbb{R}\right\}$ is characterized by the commutative algebra $\mathscr{M}$ of observables generated by the spectral family $\left\{P_{t} \mid t \in \mathbb{R}\right\}$ of projection operators $P_{t}$. Since $V_{\lambda}=e^{-i \lambda T} \in \mathscr{M}$, the time operator $T$ is an unbounded observable associated to the algebra of observables. In contrast, the bounded functions of the generator $\Lambda$ of the time evolution do not belong to $\mathscr{M}$ so that $\Lambda$ cannot be considered as an observable. In particular, $\Lambda$ does not represent the energy.

To summarize: To a weakly stationary process one can associate a time operator if and only if the process is purely nondeterministic in the linear sense. If the process is represented by commutative dynamical $\mathrm{W}^{*}$-system, the time operator is an unbounded observable associated to the commutative algebra of observables. The generator of the time evolution is canonically conjugated to the time operator but it is not an observable.

\subsection{FORWARD NONDETERMINISTIC PROCESSES AND NONANTICIPATING LINEAR FILTERS}

In communication theory, certain messages can be represented by a continuous stationary process. Let $\left\{f_{t} \mid t \in \mathbb{R}\right\}$ be a complex-valued weakly stationary process with zero mean and covariance

$$
C_{f}(t):=\mathcal{E}\left\{f_{t}^{*} f_{0}\right\}=\int_{-\infty}^{\infty} e^{i \lambda t} d F_{f}(\lambda) .
$$

Suppose the signal $t \mapsto f_{t}$ passes through a time-invariant, linear filter with the response function $R: \mathbb{R} \rightarrow \mathbb{R}$. Then the output of the filter is the zero mean process $t \mapsto g_{t}$

$$
g_{t}=\int_{-\infty}^{\infty} R(t-s) f_{s} d s
$$

with the covariance

$$
C_{g}(t):=\mathcal{E}\left\{g_{t}^{*} g_{0}\right\}=\int_{-\infty}^{\infty} e^{i \lambda t} d F_{g}(\lambda)
$$

The spectral distribution functions of the input and output processes are related via the frequency-response function $H$ by

$$
d F_{g}(\lambda)=|H(\lambda)|^{2} d F_{f}(\lambda) \quad, \quad H(\lambda):=\int_{-\infty}^{\infty} e^{i \lambda t} R(t) d t
$$

According to the Wiener-Krein criterion, the output process $t \mapsto g_{t}$ is purely nondeterministic in the linear sense if $\lambda \mapsto F_{g}(\lambda)$ is absolutely continuous and if

$$
\int_{-\infty}^{\infty} \frac{\left|\ln \left\{\rho_{g}(\lambda)\right\}\right|}{1+\lambda^{2}} d \lambda<\infty \quad, \quad \rho_{g}(\lambda):=\frac{d F_{g}(\lambda)}{d \lambda} .
$$

The relation 


$$
\int_{-\infty}^{\infty} \frac{\ln \left\{d F_{g}(\lambda) / d \lambda\right\}}{1+\lambda^{2}} d \lambda=\int_{-\infty}^{\infty} \frac{\ln |H(\lambda)|^{2}}{1+\lambda^{2}} d \lambda+\int_{-\infty}^{\infty} \frac{\left.d F_{f}(\lambda) / d \lambda\right\}}{1+\lambda^{2}} d \lambda
$$

implies that the output process $t \mapsto g_{t}$ is purely nondeterministic in the linear sense if and only if the input process $t \mapsto f_{t}$ is purely nondeterministic in the linear sense and the linear filter satisfies the Paley-Wiener criterion. In particular, the output process is forward purely nondeterministic in the linear sense if and only if the input process is forward purely nondeterministic in the linear sense if and only if the input process is forward purely nondeterministic in the linear sense and the linear filter is nonanticipating.

The basic stochastic process is white noise. It is defined as the generalized derivative of the Wiener process $t \mapsto w_{t}$ with incremental covariance $\mathcal{E}\left\{d w_{t} d w_{t}\right\}=d t$. The Wiener process is a mathematically rigorous model for the Brownian motion $t \mapsto B_{t}$ in one dimension whose mean squared displacement $\left\langle B_{t}^{2}\right\rangle$ has a linear dependence on time $t,\left\langle B_{t}^{2}\right\rangle=2 D t$, where $D$ is the diffusion constant. White noise $t \mapsto n_{t}=d w_{t} / d t$ is a generalized weakly stationary real-valued process with zero mean whose power spectral density is a constant at all frequencies

$$
\mathcal{E}\left\{n_{t}\right\}=0 \quad, \quad C_{n}(t-s):=\mathcal{E}\left\{n_{t} n_{s}\right\}=\delta(t-s) \quad, \quad d F_{n}(\lambda) / d \lambda=1 / 2 \pi .
$$

If the input process is white noise $t \mapsto n_{t}$, the output process $t \mapsto g_{t}$ has an absolutely continuous spectral distribution function with the density

$$
\rho_{g}(\lambda)=\frac{1}{2 \pi}|H(\lambda)|^{2},
$$

and satisfies the Wiener-Krein criterion.

For a given covariance function $t \mapsto C_{g}$ with the spectral density $\lambda \mapsto \rho_{g}(\lambda)$

$$
C_{g}(t)=\int_{-\infty}^{\infty} e^{i \lambda t} \rho_{g}(\lambda) d \lambda
$$

one can always find a linear input-output system with a response function $R: \mathbb{R} \rightarrow \mathbb{R}$, such that the response to a white noise input is a real-valued weakly stationary process $t \mapsto g_{t}$ with the covariance $C_{g}(t-s)=\mathcal{E}\left\{g_{t} g_{s}\right\}$. But this representation is far from unique. The only condition one has to satisfy is

$$
\rho_{g}(\lambda)=\frac{1}{2 \pi}|H(\lambda)|^{2} \text { with } H(\lambda):=\int_{-\infty}^{\infty} e^{i \lambda t} R(t) d t .
$$

If the Paley-Wiener criterion is met, the function $\lambda \mapsto|H(\lambda)|^{2}$ can be factorized. With this, the spectral density $\rho_{g}$ can be factorized into a boundary value of an analytic function which is holomorphic in the open upper half-plane and a boundary value of an analytic function which is holomorphic in the open lower half-plane. If we choose a filter of minimum phase type, these two functions are uniquely given by

$$
H^{ \pm}(\lambda)=\lim _{\varepsilon \downarrow 0} \exp \frac{1}{\pi i} \int_{-\infty}^{\infty} \frac{1+s(\lambda \pm i s \varepsilon)}{s-(\lambda \pm i s \varepsilon)} \frac{\left|\ln \rho_{g}(\lambda)\right|}{1+(\lambda \pm i s \varepsilon)^{2}} d s \quad, \quad \lambda \in \mathbb{R},
$$

so that the canonical Wiener factorization of the spectral density is given by

$$
2 \pi \rho_{g}(\lambda)=H^{+}(\lambda) H^{-}(\lambda)=\left|H^{+}(\lambda)\right|^{2}=\left|H^{-}(\lambda)\right|^{2}, \quad \lambda \in \mathbb{R} .
$$


Corresponding to these two canonical solutions, we get two moving average representations: The canonical backward moving average representation of a forward purely nondeterministic process

$$
\begin{aligned}
t & \mapsto g_{t}^{+}, \\
g_{t}^{+} & =\int_{-\infty} R^{+}(t-s) d w_{s} \quad, \quad R^{+}(t):=\frac{1}{2 \pi} \int_{-\infty}^{\infty} e^{-i \lambda t} H^{+}(\lambda) d \lambda \quad, \quad \mathcal{E}\left\{g_{t}^{+} g_{s}^{+}\right\}=C_{g}(t-s),
\end{aligned}
$$

and the canonical forward moving average representation of backward purely nondeterministic process $t \mapsto g_{t}^{-}$,

$$
g_{t}^{-}=\int_{t}^{\infty} R^{-}(t-s) d w_{s} \quad, \quad R^{-}(t):=\frac{1}{2 \pi} \int_{-\infty}^{\infty} e^{-i \lambda t} H^{-}(\lambda) d \lambda \quad, \quad \mathcal{E}\left\{g_{t}^{-} g_{s}^{-}\right\}=C_{g}(t-s)
$$

Since these stochastic processes are ergodic, the functions $t \mapsto g_{t}^{+}$and $t \mapsto g_{t}^{-}$can also be interpreted individually if the function $t \mapsto w_{t}$ is understood as the individual Wiener process.

To summarize: A nonanticipating time-invariant, linear filter transforms forward purely nondeterministic input-processes into forward purely nondeterministic output-processes. Every weakly stationary process which is forward purely nondeterministic in the linear sense can be realized as the output of a nonanticipating linear filter with a white-noise input. The corresponding response function can be constructed by a Wiener factorization of the spectral density of the process.

\subsection{Conclusion}

The representation of facts requires the distinction of past and future. In classical physics it is possible to break the time-reversal symmetry of infinite conservative and reversible Hamiltonian systems. The resulting irreversible and dissipative behavior can be described by nonanticipative measuring instruments, or equivalently by forward purely nondeterministic stochastic processes. Both can be characterized by the Paley-Wiener criterion. The growth of the set of facts can be described with the time operator of the associated K-flow.

\section{THE REPRESENTATION OF FACTS IN QUANTUM PHYSICS}

\section{I Quantum K-Flows}

In classical physics K-flows are the paradigmatic model for the emergence of one-sided time evolutions from a bidirectionally deterministic dynamics. The definition of a K-flow appropriate for classical physics can be generalized to quantum physics by replacing in the algebraic definition the commutative algebra by a noncommutative one. Let $\left(\mathscr{M},\left\{\alpha_{t} \mid t \in \mathbb{R}\right\}\right)$ be a dynamical $\mathrm{W}^{*}$-system consisting of a (in general noncommutative) $\mathrm{W}^{*}$-algebra $\mathscr{M}$ and a one-parameter group $\left\{\alpha_{t} \mid t \in \mathbb{R}\right\}$ of automorphisms $\alpha_{t}$. The dynamical $W^{*}$-system $\left(\mathscr{M},\left\{\alpha_{t} \mid t \in \mathbb{R}\right\}\right)$ is called a

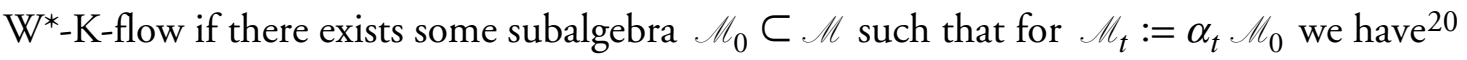

- $\mathscr{M}_{t} \subset \mathscr{M}_{s}$ for every $t<s$,

20 Emch (1976). Emch's requirement of an invariant state is no longer called for. Compare for example Narnhofer \& Thirring (1990); Benatti (1993), p. 129. 


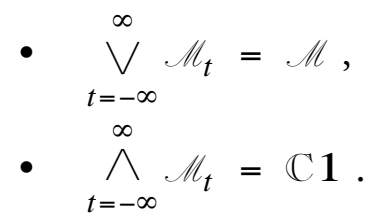

Here $\vee \mathscr{M}_{t}$ is the smallest $\mathrm{W}^{*}$-subalgebra of $\mathscr{M}$ which contains all $\mathscr{M}_{t}$, while $\wedge \mathscr{M}_{t}$ is the largest $\mathrm{W}^{*}$-subalgebra of $\mathscr{M}$ which is contained in all $\mathscr{M}_{t}$.

Conclusion: The classical concept of K-flows representing forward purely nondeterministic processes can be generalized to noncommutative irreversible quantum processes. In contrast to the classical case, noncommutative $\mathrm{K}$-systems are not sufficient to represent facts. The reason is that a representation of facts requires not only the existence of nonanticipative processes but also the impossibility of coherent superposition of facts. In other words, facts have to be represented by observables which commute with all observables of the system.

\subsection{Classical observables, Disjoint states AND PROPER MiXTUReS}

Nontrivial observables which commute with all observables are called classical observables. While in von Neumann's codification of traditional quantum theory there are no classical observables. ${ }^{21}$ Algebraic quantum mechanics allows to discuss classical observables from a fundamental point of view. All examples of rigorous derivations of classical observables refer to systems with infinitely many degrees of freedom. For example, it has been shown that in the thermodynamic limit the observables for temperature, the chemical potential and the order parameters for ferromagnets, superfluids and superconductors are classical observables.

The classical behavior of a quantum system is mirrored by the center of the algebra of observables. The center $\mathscr{L}(\mathscr{M})$ of a W*-algebra $\mathscr{M}$ consists of all elements of $\mathscr{M}$ which commute with every element of $\mathscr{M}$,

$$
\mathscr{L}(\mathscr{M}):=\{Z \mid Z \in \mathscr{M}, Z M=M Z \text { for every } M \in \mathscr{M}\}
$$

The center $\mathscr{L}(\mathscr{M})$ is a commutative $\mathrm{W}^{*}$-algebra. If the center consists only of the multiples of the identity, it is called trivial, $\mathscr{L}(\mathscr{M})=\mathbf{1} \mathbb{C}$. A W*-algebra is commutative if it is identical with its center, $\mathscr{M}=\mathscr{L}(\mathscr{M})$. If the center of the algebra of observables is trivial, then the corresponding physical system is called a pure quantum system. If the algebra of observables is commutative, then the physical system is called classical. In general, physical systems are partially quantal and partially classical. That is, the algebra of observables is noncommutative and its center is nontrivial. The nontrivial selfadjoint operators of the center are called classical observables. If $\mathscr{M}$ describes a quantum system, then $\mathscr{L}(\mathscr{M})$ describes its classical part. The classical part of a quantum system fulfills all requirements of a system of classical physics, nevertheless all its quantities intrinsically depend on Planck's constant $\hbar$.

The central part of a quantum system allows an important classification of states. The support $S_{\rho}$ of a normal state $\rho$ of a $\mathrm{W}^{*}$-algebra $\mathscr{M}$ is defined as the smallest projection operator $S \in \mathscr{M}$ such that $\rho(S)=1$. The central support $C_{\rho}$ of a state $\rho$ is defined as the smallest projection operator $C \in \mathscr{L}(\mathscr{M})$ such that $\rho(C)=1$. A state $\rho$ on the $\mathrm{W}^{*}$-algebra $\mathscr{M}$ is pure if its support $S_{\rho}$ is an atom. A state $\rho$ is called a factor state if its central support is an atom. Two states $\rho$ and $\varphi$ are called orthogonal if their supports $S_{\rho}$ and $S_{\varphi}$ are orthogonal, $S_{\rho} S_{\varphi}=0$. Two states $\rho$ and $\varphi$ are

21 This is the irreducibility postulate introduced by vonNeumann (1932) which implies that all selfadjoint operators acting on the Hilbert space of state vectors are observabless. However, we know empirically that von Neumann's irreducibility postulate is not valid in general. 
called disjoint if their central supports $C_{\rho}$ and $C_{\varphi}$ are orthogonal, $C_{\rho} C_{\varphi}=0$. Disjointness implies orthogonality but only in commutative algebras orthogonality implies disjointness. In general, disjointness is a much stronger condition than orthogonality.

A factor state $\rho$ is distinguished by the fact that it is dispersion-free with respect to every classical observable,

$$
\rho\left(Z^{2}\right)=\{\rho(Z)\}^{2} \text { for every } Z=Z^{*} \in \mathscr{Z}(\mathscr{M}) .
$$

If two states $\rho$ and $\varphi$ are disjoint, then there exists a classical observable $Z \in \mathscr{Z}(\mathscr{M})$ such that $\rho(Z) \neq \varphi(Z)$, so that disjoint states can be distinguished and classified in a classical manner. Two normal pure states $\rho$ and $\varphi$ are called equivalent if $\rho(Z)=\varphi(Z)$ for all classical observables $Z \in \mathscr{L}(\mathscr{M})$. Every state can be decomposed uniquely into a sum or an integral of disjoint factor states. ${ }^{22}$ This so-called central decomposition represents the finest unique decomposition of a nonpure state into disjoint factor states.

In contrast to the classical case, for quantum systems the convex set of all states is not a simplex, hence a convex decomposition of a nonpure factor state is never unique. ${ }^{23}$ A nonpure factor state $\rho_{\alpha}$ has infinitely many different decompositions into a convex sum of pure states. Such decompositions into pure states cannot be interpreted as a proper mixture.

If we make a classical mixture of two components (like a mixture of water and alcohol), then we tacitly presuppose that we can distinguish operationally between the two components. It makes no sense to speak of mixing indistinguishable entities. That is, it must be possible to label every component of a proper mixture so that the components can be distinguished. Since such a label must be determinable together with any other property of the component, so it has to be characterized by a value of a classical observable.

The nonpurity of factor states cannot originate in some kind of mixing, it is always due to Einstein-Podolsky-Rosen correlations of the system considered with its environment. Nonpure quantum states can be interpreted in terms of a proper mixture of pure states if and only if these pure states are mutually disjoint. ${ }^{24}$ The finest decomposition possible which allows an ignorance interpretation is the central decomposition.

Conclusions: Disjoint states are of crucial importance as final states in any processes - natural processes or measurement processes - which produce facts. This is decisive for a proper discussion of the notorious measurement problem of quantum theory. The measurement problem is not - as often asserted - the problem how a pure state can be transformed into a nonpure state, or how the density operator can become diagonal in a preferred basis. This is a trivial task - appropriate dynamical linear semigroups and their Hamiltonian dilations can describe such a decoherence mechanism. A proper statistical description of the measurement process has to show that the dynamics of an isolated quantum system can create facts. That is, one has to show that there exists a dynamics which transforms factor states into a classical mixture of disjoint factor states.

\subsection{THE EMERgenCE OF FACTS IN QUANTUM SYSTEMS}

Classical observables and disjoint states exist only if the quantum system has infinitely many degrees of freedom. If the quantum system is coupled to the electromagnetic radiation field, there

22 For details, compare for example Takesaki (1979), chapt.IV.6.

23 The state space of a $C^{*}$-algebra $\mathscr{C}$ is a simplex if and only if $\mathscr{C}$ is commutative. Compare Takesaki (1979), p. 251 .

24 Compare also Amann \& Primas (1996). 
are always infinitely many inequivalent representations, so that in this case the existence of many disjoint states is the rule. Accordingly, there are many quantum systems with classical observables which can be used to represent facts. The creation of new facts by a dynamical system, however, is a hard nut to crack: a measurement process is a dynamical process which has to transform a factor state into disjoint final factor states. A very general result due to Klaus Hepp shows that such a process cannot be described by any automorphism of the algebra of observables:

\section{No-go theorem for any automorphic dynamics ${ }^{25}$}

If $\rho$ and $\varphi$ are two disjoint states on a $\mathrm{C}^{*}$-algebra and if $\alpha$ is an automorphism of this $C^{*}$-algebra, then the transformed states $\rho \circ \alpha$ and $\varphi \circ \alpha$ are disjoint.

This theorem shows also a crucial difference between commutative and noncommutative K-flows: A classical commutative K-flow maps equivalent states into disjoint states while a noncommutative K-flow cannot change the equivalence class. To the best of my knowledge, there are just two reasonable ways out of this situation:

- The fundamental dynamics is invariant under time-reversal but not given by a oneparameter group of automorphisms.

- The fundamental dynamics is given by a one-parameter group of automorphisms but the disjointness of the final states is reached only asymptotically.

I will not consider the first possibility because no general theory is available for nonautomorphic time evolution invariant under time-reversal. But I would like to stress that the postulate of an automorphic dynamics has no sound physical basis. Many physically reasonable $\mathrm{C}^{*}$-algebraic systems without an automorphic dynamics are known.

The second point of view has been introduced by Hepp (1972). He proved the important result hat for appropriate quantum systems there exists a one-parameter automorphism group $\left\{\alpha_{t} \mid t \in \mathbb{R}\right\}$ such that for equivalent initial states $\rho_{1}, \rho_{2}, \ldots$ the asymptotic limits $\rho_{1} \circ \alpha_{t}$, $\rho_{2} \circ \alpha_{t}$ exist for $t \rightarrow \infty$ and are disjoint. Nevertheless, as shown by John Bell (1975), under Hepp's assumptions alone the measurement process is not well-posed since it is still possible to undo the measurement for any finite time by a quasilocal perturbation. As shown by Lockhart $\&$ Misra (1986), the key to the final resolution lies in recognizing the measurement apparatus as an irreversible dynamical system which breaks the time-reversal symmetry in such a way that it acts as a nonanticipative system.

In order to incorporate the irreversible and nonanticipative behavior of actual measurement apparatuses into the theory, one has to select a W*-algebra $\mathscr{M}_{0} \subset \mathscr{M}$ of observables which one can observe with a nonanticipative laboratory instrument. The requirement of nonanticipativity means that $\mathscr{M}_{t} \subseteq \mathscr{M}_{s}$ for $t<s$, where $\mathscr{M}_{t}:=\alpha_{t} \mathscr{M}_{0}$. In terms of normal conditional expectations $\mathscr{E}_{t}:=\alpha_{t} \mathscr{E}_{0} \alpha_{-t}$ with $\mathscr{E}_{0}(\mathscr{M})=\mathscr{M}_{0}$, one gets $\mathscr{E}_{t} \leq \mathscr{E}_{s}$ for $t<s$. The family $\left\{\mathscr{E}_{t} \mid t \in \mathbb{R}\right\}$ is either constant (Hepp's case, where the time reversal symmetry holds), or increasing as $t$ increases (so that the time-reversal symmetry is broken). In the case of broken time-reversal symmetry, the automorphism group $\left\{\alpha_{t}\right\}$ and the $\mathrm{W}^{*}$-algebra $\mathscr{M}_{0}$ give rise to a generalized K-flow. The appropriate tools for the discussion of noncommutative K-flows are again the Wiener factorization and the theory of Hardy spaces.

Such symmetry-breaking K-flows cannot be classical and they do not generate classical observables in any finite time. However, they do produce asymptotically disjoint final states. That is, the 
emergence of disjoint states describing facts is gradual. It occurs progressively over finite amounts of time. The exact disjointness is reached only in the limit $t \rightarrow \infty$ but the objective irreversibility of the K-flow warrants that the process cannot be undone. In this sense we can speak of approximately disjoint states and approximate facts even for finite times.

In everyday life we usually idealize approximate facts. For example, the event of death is considered as a fact. It is characterized by the irreversible loss of the bodily attributes and functions that constitute life. This is a continuous physiological process which cannot be undone. Although this process can proceed very quickly, there is, however, no definite instant of death.

In this sense, the Hepp-Lockhard-Misra-process is a very reasonable model for the emergence of facts. It also shows that the so-called "measurement problem of quantum mechanics" is neither a pseudoproblem nor a philosophical question. It is a well-posed problem of mathematical physics which can be discussed in the framework of algebraic quantum mechanics, provided we take the nonanticipative character of all laboratory instruments into account.

Conclusion: If we include the whole environment of an open quantum system and if we describe the resulting system by an automorphic dynamical $C^{*}$-system, then this system cannot generate new facts in finite time. However such system can generate in a strictly irreversible manner asymptotically disjoint final states which are described by noncommutative K-flows. Every K-flow has its own typical relaxation time. For finite times much larger than the relaxation time asymptotically disjoint states describe approximate facts which corresponds to the facts of everyday life.

\section{ACKNOWLedgment}

I thank Harald Atmanspacher for many discussions about the topics presented in this article.

\section{REFERENCES}

Amann, A. \& Primas, H. (1996): What is the referent of a nonpure quantum state? Experimental Metaphysics Quantum Mechanical Studies in Honor of Abner Shimony. In press.

Bell, J. S. (1975): On wave packet reduction in the Coleman-Hepp model. Helv. Phys. Acta 48, 93-98.

Benatti, F. (1993): Deterministic Chaos in Infinite Quantum Systems. Berlin: Springer.

Cornfeld, I. P., Fomin, S. V. \& Sinai, Ya. G. (1982): Ergodic Theory. New York: Springer.

Costa de Beauregard, O. (1987): Time, the Physical Magnitude. Dordrecht: Reidel.

Cramér, H. (1961): On some classes of non-stationary stochastic processes. In: J. Neyman (ed.): Proceedings of the Fourth Berkeley Symposium on Statistics and Applied Probability. Volume II: Contributions to Probability Theory. Berkeley: University of California Press. Pp. 57-78.

Cramér, H. (1961): On the structure of purely non-deterministic stochastic processes. Ark. Mat. 4, 249-266.

Cramér, H. \& Leadbetter, M. R. (1967): Stationary and Related Stochastic Processes. New York: Wiley.

Einstein, A. (1909): Zum gegenwärtigen Stand des Strablungsproblems. Physikalische Zeitschrift 10, 185-193.

Emch, G. G. (1976): Generalized K-Flows. Commun. Math. Phys. 49, 191-215.

Feynman, R. P. \& Hibbs, A. R. (1964): Quantum Mechanics and Path Integrals. New York: McGraw-Hill.

Fock, V.A. (1957): On the interpretation of quantum mechanics. Czechosl. J. Phys. 7, 643-656. Russian original: Usp. Fiz. Nauk 62, 461(1957).

Gustavson, K. \& Misra, B. (1976): Canonical commutation relations of quantum mechanics and stochastic regularity. Letters in Mathematical Physics 1, 275-280.

Hanner, O. (1950): Deterministic and non-deterministic stationary random processes. Ark. Mat. 1, 161-177. 
Havas, P. (1965): Relativity and causality. In: Y. Bar-Hillel (ed.): Logic, Methodology and Philosophy of Science. Amsterdam: North-Holland. Pp.347-362.

Hepp, K. (1972): Quantum theory of measurement and macroscopic observables. Helv. Phys. Acta 45, 237-248.

Kakutani, S. (1950): Review of "Extrapolation, Interpolation and Smoothing of Stationary Time Series" by Norbert Wiener. Bull. Amer. Math. Soc. 56, 378-381.

Kalman, R. E., Falb, P. L. \& Arbib, M. A. (1969): Topics in Mathematical System Theory. New York: McGraw-Hill.

Khintchine, A. (1934): Korrelationstheorie der stationären stochastischen Prozesse. Mathematische Annalen 109, 604615.

Kolmogoroff, A. (1933): Grundbegriffe der Wahrscheinlichkeitsrechnung. Berlin: Springer.

Krein, M. G. (1945): On a generalization of some investigations of G. Szegö, W.M. Smirnov, and A.N. Kolmogorov. Dokl. Akad. Nauk SSSR 46, 91-94, [in Russian].

Krein, M. G. (1945): On a problem of extrapolation of A.N. Kolmogorov. Dokl. Akad. Nauk SSSR 46, 306-309, [in Russian].

Krengel, U. (1971): K-flows are forward deterministic, backward completely non-deterministic stationary point processes. J. Math. Anal. Appl. 35, 611-620.

Krengel, U. (1973): Recent results on generators in ergodic theory. Transactions of the Sixth Prague Conference on Information Theory, Statistical Decision Functions, Random Processes. Prague: Academia. Pp.465-482.

Lockhart, C. M. \& Misra, B (1986): Irreversibility and measurement in quantum mechanics. Physica A 136, 47-76.

Narnhofer, H. \& Thirring, W. (1990): Algebraic K-systems. Letters in Mathematical Physics 20, 231-250. Errata: Letters in Mathematical Physics, 22, 81 (1991).

Neumann, J. von (1932): Mathematische Grundlagen der Quantenmechanik. Berlin: Springer.

Paley, R. E. A. C. \& Wiener, N. (1934): Fourier Transforms in the Complex Domain. Providence: American Mathematical Society.

Pfaffelhuber, E. (1971): Generalized impulse response and causality. IEEE Transactions on Circuit Theory CT-18, $218-223$.

Picci, G. (1986): Application of stochastic realization theory to a fundamental problem of statistical physics. In: C. I. Byrnes \& A. Lindquist (eds.): Modelling, Identification and Robust Control. Amsterdam: North-Holland. Pp. 211-258.

Picci, G. (1988): Hamiltonian representation of stationary processes. In: I. Gohberg, J. W. Helton \& L. Rodman (eds.): Operator Theory: Advances and Applications. Basel: Birkhäuser. Pp.193-215.

Rosenblatt, M. (1971): Markov Processes: Structure and Asymptotic Behavior. Berlin: Springer.

Scarpellini, B. (1979): Entropy and nonlinear prediction. Z. Wahrscheinlichkeitstheorie verw. Gebiete 50, 165-178.

Scarpellini, B. (1979): Predicting the future of functions on flows. Math. Systems Theory 12, 281-296.

Synge, J. L. (1960): Classical dynamics. In: S. Flügge (ed.): Principles of Classical Mechanics and Field Theory. Encylopedia of Physics. Voulme III/1. Berlin: Springer. Pp.1-225.

Takesaki, M. (1979): Theory of Operator Algebras I. New York: Springer.

Tjøstheim, D. (1975): Multiplicity theory for multivariate wide sense stationary generalized processes. J. of Multivariate Analysis 5, 314-321.

Tjøstheim, D. (1976a): A commutation relation for wide sense stationary processes. SIAM J. Appl. Math. 30, 115-122.

Tjøstheim, D. (1976b): Spectral generating operators for non-stationary processes. Adv. Appl. Prob. 8, 831-846.

Wiener, N. (1923): Differential space. Journal of Mathematics and Physics (MIT) 2, 131-174.

Wiener, N. (1930): Generalized harmonic analysis. Acta Math. 55, 117-258.

Wiener, N. (1942): Extrapolation, Interpolation, and Smoothing of Stationary Times Series, with Engineering Applications. Report to Section D2, National Defence Research Committe (NDRC). Issued as Classified Report in February 1942

Wiener, N. (1949): Extrapolation, Interpolation, and Smoothing of Stationary Times Series. With Engineering Applications. New York: MIT Technology Press and Wiley.

Wold, H. (1938): A Study in the Analysis of Stationary Times Series. Stockholm: Almquist and Wiksell. 